\title{
Adaptation to apparent concomitant motion in the absence of physical or retinal motion
}

\author{
JEROME D. TIETZ and WALTER C. GOGEL \\ University of California, Santa Barbara, California 93106
}

\begin{abstract}
Continued fixation of a point of light in the dark moving concomitantly with the observer's head motion has been found to lead to motion adaptation. This adaptation is shown in that, following the adaptation period, a physically stationary object viewed with head movement appears to move concomitantly with a direction and phase opposite to those of the adapting stimulus. The present study was concerned with identifying the conditions responsible for this adaptation. Of particular interest was the question of whether the concomitant motion sufficient for adaptation is a physical or an apparent motion. Two kinds of concomitant motion were used as adaptation stimuli: a point of light in the dark that appeared to move concomitantly with the observer's head motion but was physically stationary and a point that physically moved concomitantly with the head but appeared stationary. Adaptation occurred in the first but not in the second situation. This result indicates that perceived, not physical, concomitance is the condition required for adaptation.
\end{abstract}

The early studies of perceptual adaptation to inverting, reversing, or displacing lenses often reported an adaptation of the perception of motion of the visual array caused by the rearranged head-motion/opticalmotion relation. After wearing the lenses for a period of hours or days, the observer found that the visual scene tended to regain its normal stationary appearance as the head was moved (Kohler, 1964). This adaptation represents a modification of the normal position constancy which allows us to perceive stationary objects as stationary (and moving objects as moving) despite variation in the proximal stimulus caused by the motion of the observer's head.

Recent studies on the modification of position constancy have dispensed with the distorting goggles and instead used a visual target that physically moves contingent upon the observer's head motion; that is, as the observer moves his head to the right, the target consistently moves to the left, or right, or up, or in some other direction (Hay, 1971; Hay \& Goldsmith, 1973; Wallach \& Kravitz, 1965). Such stimulus motion is "concomitant" with the head motion in that the direction and velocity of the stimulus and head motion are correlated. At first, as with distorting lenses, the observer usually correctly perceives the target as moving but sees less and less motion with continued observation. In the hypothetical case of total adaptation, the target comes to appear stationary despite its physical concomitant motion. The amount of adaptation is typically measured by the size of the aftereffect; that is, following adaptation the observer moves his head and reports that a physically stationary stimulus appears to move with a phase and direction opposite to those of the adapting stimulus.

The preparation of this article was supported by PHS Research Grant MH-15651 from the National Institute of Mental Health.
What are the necessary and sufficient conditions for modification of direction constancy? Previous studies have either implied that physical concomitance was the adequate stimulus or have been ambiguous on this issue: Adaptation occurs for a "mobile target whose objective displacement was dependent on the S's head rotations" (Wallach \& Kravitz, 1965, p. 217) or when "A visual target was moved left and right in exact synchrony with vertical movements of the head.... The critical factor in the adaptation is the novel correlation of eye and head movement elicited during the period of exposure" (Hay, 1968, p. 429). The purpose of the present study is to demonstrate that such statements which tend to implicate physical concomitance as the important variable for adaptation are inadequate and that perceptual variables, particularly the perceived distance and perceived motion of the target, are necessary factors in predicting the direction and amount of adaptation.

It has been shown that the position constancy mechanism must incorporate cues that determine the perceived distance to the target. Hay and Sawyer (1969) found that increasing or decreasing the convergence angle necessary to fixate the eyes on a physically stationary point of light in the dark caused the point to appear to move respectively with or against the observer's head motion. Wallach, Yablick, and Smith (1972) found still larger effects with a simultaneous change in accommodation and convergence. Gogel and Tietz (1973) reported that if a physically stationary point of light is made to appear nearer or farther than its physical distance as a consequence of nonoculomotor distance factors (in this case the specific distance tendency), it also appears to move with or against the head, respectively. This study suggests that perceived distance rather than distance cues per se is the significant factor. The reason for this effect of perceived distance upon 

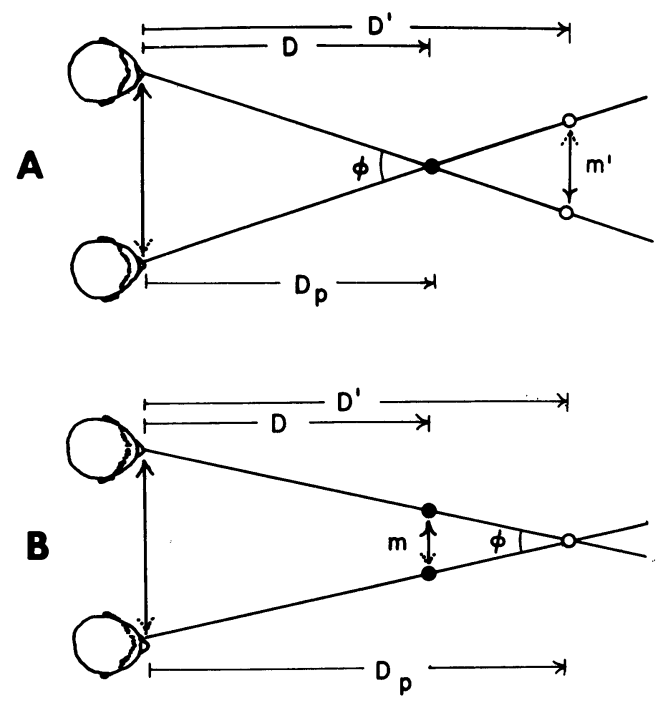

Figure 1. The two adaptation situations used in the present experiment.

position constancy is illustrated in Figure 1A in which a point of light at a physical distance $\mathrm{D}$ and perceived to be at a greater distance $\left(D^{\prime}\right)$ appears to move $\left(m^{\prime}\right)$ in a direction opposite to ("against") the motion of the head as the observer moves his head repetitively left and right. The angle through which the eyes must turn during head motion to maintain fixation on the target is $\phi$.

If a stationary object can be made to appear to move by altering its perceived distance with its physical distance kept constant, then it should be possible to arrange the physical and perceived distance in just the right combination to make an object that is physically moving concomitantly with the head appear stationary. This case is illustrated in Figure 1B, in which a point at the physical distance $D$ is made to physically move (m) in the same direction as ("with") the head. As is indicated, if the object is perceived to be at the greater distance $\left(D^{\prime}\right)$, it will not appear to move $\left(\mathrm{m}^{\prime}=0\right)$. Thus, whenever the perceived and physical distance of an object differ, the amount or direction of a physical concomitant motion does not uniquely specify the amount or direction of the apparent concomitant motion.

Returning to the topic of adaptation of position constancy, the question arises whether it is the physical or the apparent concomitant motion that is the important independent variable in the modification of position constancy by adaptation. In other words, will adaptation occur with physical concomitance in the absence of apparent concomitance (Case B of Figure 1) or with apparent concomitance in the absence of physical concomitance (Case A of Figure 1)? Previous research usually does not allow this question to be answered because the viewing conditions either provided fairly accurate distance perception such that the physical concomitance $(\mathrm{m})$ and the apparent concomitance $\left(\mathrm{m}^{\prime}\right)$ were similar or the amount of apparent concomitant motion was not measured.
The experiment reported below was designed to separate physical from perceived concomitant motion by using the two adaptation conditions shown in Figure 1. If $\mathrm{m}$ in the absence of $\mathrm{m}^{\prime}$ can determine the adaptation, adaptation should occur under the conditions illustrated by Case B but not for those illustrated by Case A. If perceived, not physical concomitance is crucial, adaptation should occur in Case A but not in B.

Position constancy occurs whenever $m=m^{\prime}=0$. It was noted that an adaptation to concomitant motion is often measured by a change in position constancy as a result of the adaptation period. That is, a physically stationary point $(m=0)$ that previously appeared stationary $\left(\mathrm{m}^{\prime}=0\right)$ as the head was moved, following adaptation, appears to move $\left(\mathrm{m}^{\prime} \neq 0\right)$ in a direction opposite to the direction of motion of the adapting stimulus. Since, as shown in Figure $1, \mathrm{~m}$ and $\phi$ are related, the adaptation also can be expressed as the change in the $\phi$ required for $\mathrm{m}^{\prime}=0$ between the pre- and postadaptation test. If the adapting stimulus moves concomitantly against the direction of the head motion, the $\phi$ required in order for $\mathrm{m}^{\prime}=0$ will increase as a result of the adaptation. If the adapting stimulus has a concomitant motion in the same direction as the motion of the head, the $\phi$ required for $\mathrm{m}^{\prime}=0$ will decrease as a result of the adaptation. Thus, in the case of Figure $1 \mathrm{~A}$, the apparent concomitant motion $\mathrm{m}^{\prime}$ is an effective stimulus for adaptation if the $\phi$ required for $\mathrm{m}^{\prime}=0$ is greater in the post- than in the preadaptation test. In the case of Figure 1B, the physical concomitant motion $m$ is an effective adaptation stimulus if $\phi$ required in order for $\mathrm{m}^{\prime}=0$ is less in the post- than in the preadaptation test. The hypothesis of the study is that, in order for $\mathrm{m}^{\prime}=0, \phi$ will increase between pre- and postadaptation test when the adaptation stimulus is that represented by $\mathrm{m}^{\prime}$ of Figure 1A but no change in $\phi$ will occur when the adaptation stimulus is that represented by $m$ of $1 B$. Such a result is evidence that apparent, not physical, concomitant motion is the adequate stimulus for adaptation of visual position constancy.

\section{METHOD}

\section{Observers}

The observers were 21 men and 27 women students taking introductory psychology (mean age $=18.8$ years). All had an uncorrected visual acuity of at least $20 / 30$ in both eyes, and all were naive as to the purpose of the experiment.

\section{Apparatus}

Consistent with the previous discussion, an apparatus was required that could vary the physical concomitant motion (m) of a point at a constant distance from the observer and therefore permit $\phi$ to be varied until the observer reported that $\mathrm{m}^{\prime}=0$. This was accomplished using the apparatus described by Gogel and Newton (1976). This apparatus uses mechanical levers to link the headrest to the point of light so that the point at a constant physical distance can be moved laterally concomitantly with the lateral motion of the head and chinrest. With this apparatus the magnitude and phase of the physical motion of the point of light (with or against the head) is continuously variable and can be adjusted by throw switches located either near the 
observer's right knee or at the experimenter's position outside the booth.

The observer sat in a lightproof booth, one wall of which contained an aperture through which the stimuli were viewed monocularly with the right eye. During the observations, the observer placed his head in a head-and chinrest which was easily moveable on ball-bearing rollers through a lateral distance of $10.5 \mathrm{~cm}$. The experimenter and observer communicated by means of microphones and headphones. Before the stimuli were presented, the observer was instructed to move his head repetitively from one lateral extreme to the other in time with an audible metronome click every $1.7 \mathrm{sec}$. A small sliding shutter mounted on the headrest occluded the left eye, and a larger shutter at the viewing aperture was used to control the presentations of the stimuli.

The stimulus was a point of light presented in a totally dark visual field. The light, always physically at a constant distance of $33 \mathrm{~cm}$ from the observer's eyes and at eye level, was produced by placing a $.2-\mathrm{mm}$ pinhole aperture in front of a small incandescent lamp. The point was in the observer's midsagittal plane when the head was at the midpoint of its motion.

Producing the adaptation situations of Figure 1. The decision to place the point of light $33 \mathrm{~cm}$ from the observer was based upon previous research which indicated that a monocularly observed point of light in the dark located at a distance considerably less than several meters appeared more distant than its physical distance. This specific distance tendency (SDT) occurs because an object in the absence of distance cues tends to appear at a distance of about $2 \mathrm{~m}$ from the observer regardless of its physical distance (Gogel \& Tietz, 1973). Since the point of light was at $33 \mathrm{~cm}$, it was expected from the SDT that it would appear to be more distant than its physical distance, as required by Figure 1A. Under these conditions, as indicated by Figure 1A, the point of light should appear to move opposite to the direction of the head as the head is moved laterally. This same physical distance of the point of light can also provide the conditions for the adaptation situation of Figure 1B. The case of Figure 1B was achieved by asking the observer to adjust the switch that physically moved the point of light concomitant with the head motion until the point of light no longer appeared to move. Since the apparent distance of the point of light was greater than its physical distance, this adjustment, as illustrated in Figure 1B, resulted in a physical concomitant motion $(\mathrm{m})$ in the same direction as the motion of the head.

\section{Procedure}

Before entering the booth, the observer was shown a model to demonstrate apparent concomitant motion "with" and "against" the head. In the booth the observer's task was to reduce the apparent motion of the point of light by throwing the switch forward and backward until the point appeared stationary as the head was moved laterally in the head- and chinrest.

The basic design of the study was to present a pretest in which the observer, while moving his head, used the throw switch to adjust $\mathrm{m}$, and thus $\phi$, until the apparent motion $\left(\mathrm{m}^{\prime}\right)$ of the point of light was reduced to zero. Following this null adjustment, one of two adaptation conditions illustrated in Figure 1 was presented for $5 \mathrm{~min}$, during which time the observer moved his head right and left while fixating the point of light. Following the adaptation period, a posttest was given in which the observer again adjusted $\phi$ until $\mathrm{m}^{\prime}=0$. Differences between $\phi$ obtained on the pre- and posttest measured the amount of perceptual adaptation.

The procedural details for each trial were as follows. Before each trial the experimenter adjusted the throw switch randomly in either direction to insure that the observer initially perceived motion as he moved his head in the head- and chinrest. In the pretest the booth lights were turned off, the metronome was turned on, and the observer began moving his head left and right in time with the clicks. The shutter was then opened and using the switch the observer adjusted $\phi$ until the point of light no longer appeared to move. In this adjustment a bracketing procedure was used; that is, if the observer initially saw motion of a particular phase (with or against the head), the switch was held in a specified direction until the point of light appeared clearly to move in the other phase (against or with the head). The switch was then adjusted back and forth as necessary to achieve the setting of no apparent motion. After the pretest the shutter was closed and the booth lights were turned on for $20 \mathrm{sec}$.

The adaptation condition in Case A consisted of the situation shown in Figure 1A, in which the point of light at $33 \mathrm{~cm}$ was physically stationary as the head moved laterally. The adaptation condition in Case B consisted of the situation shown in Figure 1B, in which the point of light at $33 \mathrm{~cm}$ physically moved concomitant with the head motion, as defined by the $\phi$ adjustment, producing no apparent motion in the pretest trial. With either adaptation condition, the observer fixated the stimulus for $5 \mathrm{~min}$ while moving his head in the head-and chinrest. At the beginning and end of the 5-min period, the observer verbally reported (1) the apparent distance to the point in feet or inches or some combination of both, (2) whether the point appeared stationary or appeared to move with or against the head, and (3) if apparent motion occurred, the distance the point appeared to move from one extreme to the other in feet or inches or some combination of both.

The shutter was then closed and the observer kept moving his head for $20 \mathrm{sec}$ with the booth lights off. Before opening the shutter to begin the posttest, the experimenter adjusted the switch to duplicate the observer's pretest setting that resulted in the perception of the point as stationary. If adaptation had occurred during the 5-min period, the observer would be expected to see the point move with the predicted phase in the posttest. To measure the amount of adaptation (the pretestposttest change in $\phi$ necessary to perceive the point as stationary), the observation shutter was opened and the observer, continuing to move his head, verbally reported whether the point appeared to move with or against his head. If no motion was reported, the trial was terminated. If motion was reported, the bracketing procedure was again used to reach a new no-motion setting of the switch.

The order of presenting Cases A and B was alternated between observers. Prior to the two experimental conditions, at the beginning of the experiment, two practice trials were given during which the observer used the bracketing technique to achieve the perception of no motion of the point. Between trials the booth lights were turned on for $3 \mathrm{~min}$ and the observer looked at objects in the booth in order to dissipate any adaptation effects possibly remaining from the preceding trial.

\section{RESULTS}

The values of $\phi$ required for the point of light to appear stationary in the pre- and posttests are shown in Table 1. The lower portion of the table shows a summary of the important characteristics that distinguish the adaptation conditions of Cases $A$ and $B$. The value of $\phi$ for the adaptation condition of Case B is an average and was determined for each observer by his adjustment in the pretest that resulted in $\mathrm{m}^{\prime}=0$. Since there was no significant difference between the median $\mathrm{D}^{\prime}$ reports obtained at the beginning and end of the adaptation period, the medians quoted below represent an average of the two values.

\section{Adaptation with Case A}

In this situation apparent, but no physical, concomitant motion was present during the adaptation period. From the $\mathrm{m}^{\prime}$ reports, all observers perceived the "adapt- 
Table 1

Conditions of Adaptation Used in the Experiment and the Amount of Eye Turning $(\phi)$ Required for the Fixated Point of Light to Appear Stationary in the Pre- and Posttests

\begin{tabular}{lccccc}
\hline & \multicolumn{3}{c}{ Monocular } & Observation \\
\cline { 2 - 3 } \cline { 2 - 3 } \cline { 5 - 6 } & \multicolumn{2}{c}{ Case A* } & & \multicolumn{2}{c}{ Case B** } \\
\cline { 2 - 3 } \cline { 5 - 6 } Mean & Pretest & Posttest & & Pretest & Posttest \\
Median & 5.63 & 6.77 & & 5.76 & 5.83 \\
SD & 5.23 & 6.01 & & 5.62 & 5.23 \\
\hline & 2.22 & 2.27 & & 1.96 & 2.30 \\
\hline
\end{tabular}

${ }^{*} D=33 \mathrm{~cm}, \phi=17.9 \mathrm{deg}, m=0, m^{\prime}$ without $m . \quad * * D=33 \mathrm{~cm}$, average $\phi=5.0$ deg, average $m=7.7 \mathrm{~cm}, m$ without $m^{\prime}$.

ing" point as moving against the head even though it was physically stationary. Although the decrease in mean $\mathrm{m}^{\prime}$ during the adaptation period, from 16.7 to $11.7 \mathrm{~cm}$, was nonsignificant $[\mathrm{t}(47)=1.57]$, the $\phi$ values increased significantly from the pre- to posttest $[t(47)=4.20]$ in the direction one would expect if the apparent motion against the head were an effective adaptation stimulus.

In order to perceive the stationary point as moving against the head, Figure 1A indicates that the observer must have perceived it to be more distant than its physical location. Although the median verbal report of the distance to the $33-\mathrm{cm}$ stimulus was $30.5 \mathrm{~cm}$, prior research (Gogel, 1977) has indicated that, under reduced cue conditions with monocular observation, perceived distance is approximately 1.7 times as great as the verbal report of distance. Therefore, a verbal report of $30.5 \mathrm{~cm}$ represents a perceived distance of approximately $52 \mathrm{~cm}$.

\section{Adaptation with Case B}

In this situation physical, but not apparent, concomitant motion was present during the adaptation period. The $\mathrm{m}^{\prime}$ reports indicated that during the 5-min adapting period, as expected, observers did not report a significant amount of motion in the adaptation stimulus, either at the beginning $[t(47)=.49]$ or at the end $[\mathrm{t}(47)=.55]$ of the 5 -min adaptation period, despite the fact that the point was moving physically with the head through an average distance of $7.7 \mathrm{~cm}$. If adaptation to this physical concomitant motion had occurred, $\phi$ would have decreased from pre-to posttest, but the results in Table 1 show no significant change $[t(47)=.23]$. Thus, the physical concomitant motion was not perceived and adaptation did not occur. The reported median $D^{\prime}$ in this condition was $30.5 \mathrm{~cm}$.

\section{DISCUSSION}

The present study represents an application of the point of view (Gogel, 1973a, 1973b) that a parsimonious list of variables upon which a perception depends often includes other perceptions (perceptual interactions). The dependent perception in this case was the perception of position as the head is moved (position constancy). The independent perception was perceived concomitant motion. Adaptation occurred only when concomitant motion was perceived, regardless of whether or not the stimulus was physically moving. It follows that if adaptation occurs with physical concomitant motion, it occurs because the physical concomitant motion has produced an apparent concomitant motion. Perceived concomitance, then, is a sufficient and perhaps a necessary condition for adaptation. There is some suggestion in the literature, however, that apparent concomitant motion is not a necessary condition for motion adaptation. Wallach, Yablich, and Smith (1972) reported that, when observers viewed normally illuminated scenes through goggles that altered convergence and accommodation, modification of position constancy resulted, but the observers were reportedly unaware of unusual field displacements during head motion. It is puzzling why no motion was reported considering the similarity to the early experiments on distorting lenses. The Wallach et al. study used a mild distortion of a natural scene with multiple cues; perhaps under these conditions, as compared with a point of light in an otherwise dark environment, the apparent concomitant motion must be extreme before it will be reported.

The conclusion that apparent concomitant motion is a sufficient and perhaps a necessary condition for motion adaptation has consequences for theories of adaptation of concomitant motion. Usually theories of motion adaptation indicate that the necessary and sufficient conditions for the adaptation are a sustained exposure to a ratio of eye turning or retinal displacement that is nonnormal for an object located at the particular distance (see Hay, 1968). However, the present experiment shows that, in defining what is "nonnormal," the perceptual variables of either or both perceived distance or perceived concomitant motion must be considered. It is surprising that, although cues of distance and possibly perceived distance have been recognized as important in position constancy (Hay \& Sawyer, 1969; Wallach, Yablick, \& Smith, 1972), in the most explicit model of position constancy modification offered so far (Hay \& Goldsmith, 1973), perceived distance and perceived motion are not discussed, nor do most experiments on adaptation of position constancy even obtain measures of these variables.

\section{REFERENCES}

Goges, W. C. The organization of perceived space: I. Perceptual interactions. Psychologische Forschung, 1973, 36, 195-221. (a)

Gogel, W. C. The organization of perceived space: II. Consequences of perceptual interactions. Psychologische Forschung, 1973, 36, 223-247. (b)

GoGEL, W. C. An indirect measure of perceived distance from oculomotor cues. Perception \& Psychophysics, 1977, 21, 3-11.

Gogel, W. C., \& Newton, R. E. An apparatus for indirect measurement of perceived distance. Perceptual and Motor Skills, 1976, 43, 295-302.

Gogel, W. C., \& TieTz, J. D. Absolute motion parallax and the specific distance tendency. Perception \& Psychophysics, 1973, 13, 284-292.

HAY, J. Visual adaptation to an altered correlation between eye movement and head movement. Science, 1968, 160, 429-430.

HAY, J. C. Does head-movement feedback calibrate the perceived direction of optical motions? Perception \& Psychophysics, 1971, 10, 286-288.

HAY, J. C., \& Goldsmith, W. Space-time adaptation of visual position constancy. Journal of Experimental Psychology, 1973, 99, 1-9.

HaY, J. C., \& SaWyer, S. Position constancy and binocular convergence. Perception \& Psychophysics, 1969, 5, 310-312.

KoHLER, I. The formation and transformation of the perceptual world. Psychological Issues, 1964, 3(4, Monograph 12), 36 .

Wallach, H., \& Kravitz, J. The measurement of the constancy of visual direction and of its adaptation. Psychonomic Science, 1965, 2, 217-218.

W Allach, H., Yablick, G., \& SMith, A. Target distance and adaptation in distance perception in the constancy of visual direction. Perception \& Psychophysics, 1972, 12, 139-145.

(Received for publication February 24, 1978.) 